\title{
GEOMETRIC PROGRAMMING WITH PROBABILISTIC DECISION VARIABLES
}

\author{
T. R. JEFFERSON and C. H. SCOTT
}

(Received 24 July 1979)

(Revised 5 May 1980)

\begin{abstract}
Here we consider a particular class of stochastic geometric programs in which the randomness occurs in the decision variables. Specifically we analyse a program in which we specify a joint normal probability for the decision variables and require the constraint set to be satisfied in the chance constrained mode. A numerical example is given to illustrate the approach.
\end{abstract}

\section{Introduction}

Uncertainty is a common feature in problems to which mathematical programming is applied. Indeed it is often assumed that the parameters are known exactly even though it is known that they are a result of statistical estimation. By this means, one eliminates the very significant technical problems that arise if the stochastic element is treated explicitly. However there are numerous applications in areas such as finance, engineering design and reliability $[11,12]$ where the stochastic element cannot be ignored. In order to cope with such problems the field of stochastic programming has evolved and considerable success has been obtained with the chance constrained approach of Charnes and Cooper [2] and recourse programming [11]. Typically these approaches allow some or all of the parameters of the mathematical program to be random variables derived from known probability density functions. In chance constrained programming, one permits constraint violations up to specified probability limits. For such problems Prekopa [8] has derived necessary and sufficient conditions for optimality for very general distributions including the normal distribution. In addition, he converts the chance constrained program into a solvable certainty equivalent.

Here we follow the chance-constrained philosophy but introduce randomness via the decision variables. We term this a mathematical program with probabilistic 
decision variables. Specifically we consider a geometric program [4] in which the decision variables are normally distributed and the linear constraint set is of the chance-constrained variety. By approximating the normal distribution we transform this into an equivalent deterministic posynomial program which may be readily solved by conventional techniques.

By a posynomial program, we mean a mathematical program of the form:

$$
\text { minimize } g_{0}(t)
$$

subject to constraints

$$
\begin{array}{ll}
g_{k}(t) \leqslant 1, & k=1,2, \ldots, p, \\
g_{k}(t) \geqslant 1, & k=p+1, \ldots, r,
\end{array}
$$

and positivity conditions

$$
t_{j}>0, \quad j=1, \ldots, m
$$

Here

$$
g_{k}(t)=\sum_{i \in[k]} \mathrm{c}_{i} \prod_{i=1}^{m} t_{j}^{a_{1},}, \quad k=0, \ldots, r,
$$

and $[k], k=0,1, \ldots, r$, is a collection of disjoint index sets which form a sequential partition of the integers 1 to $n$ ( $\mathrm{n}$ is the total number of terms in the objective function plus all the constraints). The $a_{i j}$ are arbitrary real exponents and the coefficients $c_{i}$ are positive. The name posynomial is derived from the polynomial form of the functions and the positivity of the coefficients and variables.

Posynomials arise naturally in the disciplines of economics and engineering. Moreover as polynomials may be converted into posynomials, posynomials are of wide applicability [7]. Posynomial programs form the original class of programs to be analysed by geometric programming duality which provides the basis for efficient computer codes $[3,9,10]$.

As a result of the versatility of the polynomial form for modelling and the basis for algorithms provided by geometric programming it is valuable if geometric programs with probabilistic decision variables can be converted into deterministic posynomial form. In Section 2, we carry this calculation through and give a computational example in Section 3.

\section{Certainty equivalent program}

We consider a linearly constrained posynomial program of the form:

$$
\begin{array}{ll} 
& \text { minimize } g_{0}(t) \\
\text { subject to } & \sum_{i \in[k]} c_{i} t_{j(i)} \leqslant 1, \quad k=1, \ldots, p .
\end{array}
$$


Here $j(i)$ determines the variable $j$ appearing in the $i$ th term. Although the linear constraints may appear to be a considerable restriction on the posynomial form, in many design and scheduling problems all the nonlinearity appears in the objective function. Let the variables $t_{j}$ be independent normally distributed random variables with mean $\mu_{j}$ (a variable) and variance $\sigma_{j}^{2}$ (a constant) for all $j$ that is $N\left(\mu_{j}, \sigma_{j}^{2}\right)$. Hence the left hand side of each constraint is a normal variate with mean $\sum_{\mathrm{i} \in[\mathrm{k}]} c_{i} \mu_{j(i)}$ and variance $\sum_{i \in[k]} c_{i} \sigma_{j(i)}^{2}$.

Initially we consider the problem with one constraint of the form (6). We require the probability of the left-hand side to be less than 1 with at least probability $q$. Hence

$$
P(x \leqslant 1) \geqslant q
$$

where $x$ is

$$
N\left(\sum_{i \in[k]} \mathrm{c}_{i} \mu_{j(i)}, \quad \sum_{i \in[k]} \mathrm{c}_{i} \sigma_{j(i)}^{2}\right) .
$$

Thus we seek $\mu_{j(i)}, \sigma_{j(i)}$ which minimize the objective function and such that a realization from the distribution will satisfy the constraint with probability $q$. In order to convert inequality (7) into a posynomial form we make use of a numerical approximation due to Hastings [5] who approximates the integral

$$
\frac{2}{\sqrt{ } \pi} \int_{0}^{z} \exp \left(-u^{2}\right) d u=\Phi(z)
$$

by

$$
\Phi_{1}(z)=1-1 /\left(1+b_{1} z+b_{2} z^{2}+b_{3} z^{3}+b_{4} z^{4}\right)^{4}
$$

to an accuracy of $0.5 \times 10^{-3}$ where $b_{1}=0.278393, b_{2}=0.230389, b_{3}=0.000972$, $b_{4}=0.078108$. A closer approximation is

$$
\Phi_{2}(z)=1-1 /\left(1+d_{1} z+d_{2} z^{2}+d_{3} z^{3}+d_{4} z^{4}+d_{5} z^{5}+d_{6} z^{6}\right)^{16}
$$

to an accuracy of $0.3 \times 10^{-6}$ where $d_{1}=0.0705230784, d_{2}=0.0422820123$, $d_{3}=0.0092705272, d_{4}=0.0001520143, d_{5}=0.0002765672, d_{6}=0.0000430638$.

For the purposes of this paper, $\Phi_{1}(z)$ will be employed. The use of $\Phi_{2}(z)$ is analogous.

Assuming that $q$ is close to one we have that

$$
P(x \leqslant 1)=\frac{1}{\left(2 \Pi \sum_{i \in[k]} c_{i} \sigma_{j(i)}^{2}\right)^{\frac{1}{t}}} \int_{-\infty}^{1}\left\{\exp -\left(x-\sum_{i \in[k]} c_{i} \mu_{j(i)}\right)^{2} /\left(2\left(\sum_{i \in[k]} c_{i} \sigma_{j(i)}^{2}\right)\right)\right\} d x .
$$

Setting

$$
z=\left(x-\sum_{i \in[k]} c_{i} \mu_{j(i)}\right) /\left(2 \sum_{i \in[k]} c_{i} \sigma_{j(i)}^{2}\right)^{\frac{1}{2}}, \quad P(x \leqslant 1) \geqslant q
$$


can be approximated by

$$
z \leqslant\left(1-\sum_{i \in[k]} c_{i} \mu_{j(i)}\right) /\left(2 \sum_{i \in[k]} c_{i} \sigma_{j(i)}^{2}\right)^{\frac{1}{2}}
$$

and

$$
0.5+0.5 \Phi_{1}(z) \geqslant q .
$$

Substituting equation (9) into (12), it may be rewritten in the form

$$
((1-q) /(0.5))^{t}\left(1+b_{1} z+b_{2} z^{2}+b_{3} z^{3}+b_{4} z^{4}\right) \geqslant 1
$$

We are now able to develop the certainty equivalent posynomial program for programs of the form

$$
\begin{gathered}
\text { minimize } g_{0}(E(t)) \\
\text { subject to } P\left(\sum_{i \in[1]} c_{i} t_{j(i)} \leqslant 1\right) \geqslant q .
\end{gathered}
$$

The equivalent deterministic program to $(S 1)$ is

$$
\begin{gathered}
\text { minimize } g_{0}(\mu) \\
\text { subject to } \sum_{i \in[k]} c_{i} \mu_{j(i)}+\left(2 \sum_{i \in[k]} c_{i} \sigma_{j(i)}^{2}\right)^{\frac{1}{2}} z \leqslant 1, \\
((1-q) / 0.5)^{\frac{1}{t}}\left(1+b_{1} z+b_{2} z^{2}+b_{3} z^{3}+b_{4} z^{4}\right) \geqslant 1
\end{gathered}
$$

where the variables are the scalar $z$ and vector $\mu$.

We are now in a position to consider the more interesting case of $p$ constraints where multivariate correlations play an important role. We consider the following program:

$(S p)$

$$
\begin{array}{ll} 
& \text { minimize } g_{0}(E(t)) \\
\text { subject to } & P\left(\sum_{i \in[k]} c_{i} t_{j(i)} \leqslant 1, k=1, \ldots, p\right) \geqslant q .
\end{array}
$$

In this generalization, the probability distribution is multivariate normal with mean vector (of dimension $p$ ) with components $M_{k}=\sum_{i \in[k]} c_{i} \mu_{j(i)}, k=1, \ldots, p$, and variance covariance matrix $\Sigma=\left(\sigma_{k l}\right)$ where

$$
\sigma_{k l}=\sum_{i \in[k]} \sum_{i^{\prime} \in\left[l^{\prime}\right]} c_{i} c_{i^{\prime}} \sigma_{j(i)}^{2} \delta_{j(i), j\left(i^{\prime}\right)}
$$

where $\sigma_{j}^{2}$ is the variance of the $j$ variable and $\delta_{f(i), j\left(i^{\prime}\right)}$ is a Kronecker delta.

If we define $\Delta$ to be the triangular square root of $\Sigma^{-1}$, then

$$
y=\Delta^{-1}(x-M)
$$

is $N(0, I)$ for $x, N(M, \Sigma)$, that is, the transformation removes the correlation. In order to employ the approximation $\Phi_{1}(z)$, we require the transformation $z=y / \sqrt{ } 2$ so that $x=M+\sqrt{ }(2 \Delta) z$. 
Program $(S p)$ can now be written as

$$
\begin{gathered}
\text { minimize } g_{0}(\mu) \\
\text { subject to } \sum_{i \in[k]} c_{i} \mu_{j(i)}+\sqrt{ } 2 \sum_{l=1}^{p} \Delta_{k l} z_{l} \leqslant 1, \quad k=1, \ldots, p, \\
\left(2 v_{k}\right)^{\frac{1}{2}}\left(1+b_{1} z_{k}+b_{2} z_{k}^{2}+b_{3} z_{k}^{3}+b_{4} z_{k}\right)^{4} \geqslant 1, \quad k=1, \ldots, p, \\
v_{k}+w_{k} \leqslant 1, \quad k=1, \ldots, p, \\
q \prod_{k=1}^{p} w_{k}^{-1} \leqslant 1
\end{gathered}
$$

over the variables $\mu, v, w$ and $z$. Here inequality (24) arises analogously to (17), and inequality (25) is the generalization of (18) which has been combined with (26) such that (25) is in posynomial form. Inequality (27) is the joint probability constraint which is factorizable since the correlations have been removed. If any of the $\Delta_{k l}$ are negative, equation (24) can be converted into posynomial form using the transformations found in [7].

Program $(C p)$ will always have reversed constraints (constraints of the form $\left.g_{k}(t) \geqslant 1\right)$ which means that the geometric program is not necessarily convex and hence the Kuhn-Tucker conditions are not necessary and sufficient. Prekopa [8] has shown that $(S p)$ is at least quasi-convex and the Kuhn-Tucker conditions are necessary and sufficient. The key transformation for geometric programming is the natural logarithm. Since $\partial \ln t_{j} / \partial t_{j}=1 / t_{j}>0$ for $t_{j}>0$ the transformation is nonsingular. Thus, appealing to the following theorem, Kuhn-Tucker conditions are necessary and sufficient for $(C p)$.

THEOREM. Assuming a constraint qualification holds, the Kuhn-Tucker conditions are necessary and sufficient for mathematical programs if there exists a transformation of the variable with non-zero Jacobian which transforms the program into a program for which the Kuhn-Tucker conditions are both necessary and sufficient.

Proof. Consider the mathematical program

$$
\begin{aligned}
& \min f_{0}(x) \\
& \text { subject to } f_{i}(x) \leqslant 0, \quad i=1, m
\end{aligned}
$$

for which the Kuhn-Tucker conditions are necessary and sufficient. Thus $\hat{x}$ is optimal for $(F)$ if and only if

$$
f_{i}(\hat{x}) \leqslant 0, \quad i=1, \ldots, m
$$

and there exists $\lambda_{i} \geqslant 0$ such that

$$
\lambda_{i} f_{i}(\hat{x})=0, \quad i
$$


and

$$
\nabla f_{0}(\hat{x})+\sum_{i=1}^{m} \lambda_{i} \nabla f_{i}(\hat{x})=0
$$

Let $T$ be a transformation with non-zero Jacobian of $y$ into $x$ :

$$
x=T(y) .
$$

Then $(F)$ is equivalent to

$$
\begin{aligned}
& \min f_{0}(T(y)) \\
& \text { subject to } f_{i}(T(y)) \leqslant 0, \quad i=1, \ldots, m .
\end{aligned}
$$

The Kuhn-Tucker conditions for this program are

$$
\begin{gathered}
f_{i}(T(y)) \leqslant 0, \quad i=1, \ldots, m, \\
\nabla f_{0}(T(y)) T^{\prime}(y)+\sum_{i=1}^{m} \lambda_{i} \nabla f_{i}(T(y)) T^{\prime}(y)=0
\end{gathered}
$$

or

$$
\left[\nabla f_{0}(T(y))+\sum_{i=1}^{m} \lambda_{i} \nabla f_{i}(T(y))\right] T^{\prime}(y)=0
$$

Since $T^{\prime}(y)$ is non-singular (29) holds if and only if

$$
\nabla f_{0}(T(y))+\sum_{i=1}^{m} \lambda_{i} \nabla f_{i}(T(y))=0
$$

which proves the theorem.

The example in the next section demonstrates this approach.

\section{Example}

Consider the process of making a box of length $t_{1}$, width $t_{2}$, depth $t_{3}$ subject to the constraints on $t_{1}+t_{2}+t_{3}$ being less than $140 \mathrm{~cm}$ and $t_{2}+t_{3}$ less than $80 \mathrm{~cm}$. The box which maximizes the volume is found by solving the geometric program

$$
\begin{array}{cc}
\text { minimize } & t_{1}^{-1} t_{2}^{-1} t_{3}^{-1} \\
\text { subject to } & \frac{t_{2}}{80}+\frac{t_{3}}{80} \leqslant 1 \\
\frac{t_{1}}{140}+\frac{t_{2}}{140}+\frac{t_{3}}{140} \leqslant 1
\end{array}
$$


The solution to $(V 1)$ is

$$
t_{1}=60, t_{2}=40, \quad t_{3}=40
$$

Suppose now that there is some variation in the production process so that the final dimensions are normally distributed with means $\mu_{1}, \mu_{2}, \mu_{3}$ and variances $\sigma_{1}^{2}, \sigma_{2}^{2}, \sigma_{3}^{2}$. For the example let the variances all be $\frac{1}{50}$. If the boxes exceed the specifications of $140 \mathrm{~cm}$ and $80 \mathrm{~cm}$ they must be thrown away. Suppose we do not wish to reject boxes more than $5 \%$ of the time. This problem gives the following stochastic program:

$$
\text { minimize }\left(E\left(t_{1} t_{2} t_{3}\right)\right)^{-1}
$$

subject to $P\left(\frac{t_{2}}{80}+\frac{t_{3}}{80} \leqslant 1, \frac{t_{1}}{140}+\frac{t_{2}}{140}+\frac{t_{3}}{140} \leqslant 1\right) \geqslant 0.95$

For this problem $M_{1}=\mu_{1}+\mu_{2}+\mu_{3}$ and $M_{2}=\mu_{1}+\mu_{2}$, and we have

$$
\begin{aligned}
\Sigma & =\frac{1}{50}\left(\begin{array}{ll}
2 & 2 \\
2 & 3
\end{array}\right), \\
\Delta & =\sqrt{ } 50\left(\begin{array}{cr}
\sqrt{ } 3 / 2 & -\sqrt{ } 2 / 3 \\
0 & \sqrt{ } 1 / 3
\end{array}\right) .
\end{aligned}
$$

The equivalent deterministic program is

$$
\text { minimize } \mu_{1}^{-1} \mu_{2}^{-1} \mu_{3}^{-1}
$$

subject to $10 \sqrt{ } 3 / 2 z_{1} \mu_{1}^{-1}+\frac{1}{2} \mu_{2} \mu_{1}^{-1}+\frac{1}{2} \mu_{3} \mu_{1}^{-1}+20 \mu_{1}^{-1} \leqslant 1$,

$$
\begin{gathered}
\frac{\sqrt{ } 3}{14} z_{2}+\frac{\mu_{2}}{140}+\frac{\mu_{3}}{140}+\frac{\mu_{1}}{140} \leqslant 1, \\
\frac{v_{k}^{\frac{1}{4}}}{0.5}\left(1+b_{1} z_{k}+b_{2} z_{k}^{2}+b_{3} z_{k}^{3}+b_{4} z_{k}^{4}\right) \geqslant 1, \quad k=1,2, \\
v_{k}+w_{k} \leqslant 1, \quad k=1,2, \\
0.95 w_{1}^{-1} w_{2}^{-1} \leqslant 1 .
\end{gathered}
$$

The solution to $(\mathrm{Ce})$ is

$$
\begin{gathered}
\mu_{1}=60.29, \quad \mu_{2}=39.47, \quad \mu_{3}=39.47, \\
w_{1}=0.989, \quad w_{2}=0.957 .
\end{gathered}
$$

Of interest is the fact that the setting for width and depth is reduced to 39.47 whereas the length is increased to 60.29 . 


\section{Acknowledgement}

We wish to thank Mr. Ivan Hidajat for solving the example using GPROG [6].

\section{References}

[1] M. Avriel and D. J. Wilde, "Stochastic geometric programming", in Proceedings of the Princeton Symposium on Mathematical Programming, H. W. Kuhn ed. (Princeton, New Jersey: Princeton University Press, 1970).

[2] A. Charnes, W. W. Cooper and G. H. Symonds, "Cost horizons and certainty equivalents: an approach to stochastic programming of heating oil”, Management Science 4 (1958), 235-263.

[3] R. S. Dembo, "Current state of the art of algorithms and computer software for geometric programming", J.O.T.A. 26 (1978) 149-184.

[4] R. J. Duffin, E. L. Peterson and C.Zener, Geometric programming (New York: John Wiley and Sons, 1967).

[5] C. Hastings, Approximation for digital computers (Princeton, New Jersey: Princeton University Press, 1955).

[6] T. R. Jefferson, Geometric programming with an application to transportation planning (Ph.D. Dissertation, Northwestern University, 1972).

[7] T. R. Jefferson and C. H. Scott, “Avenues of geometric programming”, N.Z. Operational Research 6 (1978), 109-136.

[8] A. Prekopa, "On probabilistic constrained programming", in Proceedings of the Princeton Symposium on Mathematical Programming, H. W. Kuhn ed. (Princeton, New Jersey: Princeton University Press, 1970).

[9] M. J. Rijckaert and X. M. Martens, "Comparison of generalized geometric programming algorithms", J.O.T.A. 28 (1978), 205-242.

[10] V. L. N. Saima, X. M. Martens, G.V. Reklaitis and M. J. Rijckaert, "A comparison of computational strategies, for geometric programs", J.O.T.A. 28 (1978), 185-204.

[11] J. K. Sengupta, Stochastic programming (Amsterdam: North-Holland, 1972).

[12] S. Vajda, Probabilistic programming (New York: Academic Press, 1972).

School of Mechanical and Industrial Engineering

University of N.S.W.

P.O. Box 1, Kensington, N.S.W. 2033

Australia 